\title{
Modeling the relationship between network operators and venue owners in public Wi-Fi deployment using non- cooperative game theory
}

\author{
Jonathan Spruytte ${ }^{1 *}$ D, Amal Benhamiche ${ }^{2}$, Matthieu Chardy², Sofie Verbrugge ${ }^{1}$ and Didier Colle ${ }^{1}$
}

\begin{abstract}
Wireless data demands keep rising at a fast rate. In 2016, Cisco measured a global mobile data traffic volume of 7.2 Exabytes per month and projected a growth to 49 Exabytes per month in 2021. Wi-Fi plays an important role in this as well. Up to 60\% of the total mobile traffic was off-loaded via Wi-Fi (and femtocells) in 2016. This is further expected to increase to $63 \%$ in 2021. In this publication, we look into the roll-out of public Wi-Fi networks, public meaning in a public or semi-public place (pubs, restaurants, sport stadiums, etc.). More concretely we look into the collaboration between two parties, a technical party and a venue owner, for the roll-out of a new Wi-Fi network. The technical party is interested in reducing load on its mobile network and generating additional direct revenues, while the venue owner wants to improve the attractiveness of the venue and consequentially generate additional indirect revenues. Three Wi-Fi pricing models are considered: entirely free, slow access with ads or fast access via paid access (freemium), and paid access only (premium). The technical party prefers a premium model with high direct revenues, the venue owner a free/freemium model which is attractive to its customers, meaning both parties have conflicting interests. This conflict has been modeled using non-cooperative game theory incorporating detailed cost and revenue models for all three Wi-Fi pricing models. The initial outcome of the game is a premium Wi-Fi network, which is not the optimal solution from an outsider's perspective as a freemium network yields highest total payoffs. By introducing an additional compensation scheme which corresponds with negotiation in real life, the outcome of the game is steered toward a freemium solution.
\end{abstract}

Keywords: Game theory, Equipment cost modeling, Public Wi-Fi, Techno-economics

\section{Introduction and definition of public Wi-Fi}

Wireless data demands keep rising at a fast rate; in 2016, Cisco measured a global mobile data traffic volume of 7.2 Exabytes per month and projected a growth to 49 Exabytes per month in 2021. The upcoming 5G standard is expected to further support this constant increase in wireless data demands. Wi-Fi plays an important role as well: up to $60 \%$ of the total mobile traffic was off-loaded via Wi-Fi (and femtocells) in 2016 and this proportion is expected to rise to $63 \%$ in 2021 [1]. These Wi-Fi networks will also play an important role in the evolution

\footnotetext{
* Correspondence: jonathan.spruytte@ugent.be

'IDLab, Department of Information Technology, Ghent University-imec,

Technologiepark-Zwijnaarde 15, 9052 Ghent, Belgium

Full list of author information is available at the end of the article
}

toward smart cities by connecting large amounts of sensors supporting new services (e.g., dynamic monitoring of waste bins).

In this publication, we will focus on public Wi-Fi networks. For the remainder of this publication, we will define a public Wi-Fi network as a wireless network that is offered to visitors of a public or semi-public place. This definition covers all sizes of networks, ranging from a coffee house with a single access point (AP) to large networks covering museums, sports stadiums, or even entire cities. We do differentiate, however, between the typical customer-facing Wi-Fi networks which are typically found in individual shops and shopping malls, and municipal Wi-Fi networks which are offered by local government to stimulate local businesses and tourism. 
At this point in time, customer-facing Wi-Fi is typically free, as business owners expect their Wi-Fi network to generate sufficient indirect revenue (e.g., by attracting additional customers or by making people stay longer) to weigh up to the costs of deploying the network.

That is not the case for city-wide municipal networks. Offering free municipal Wi-Fi has a large range of economic benefits, such as alleviating the digital divide [2] and making the region attractive to businesses and highly educated citizens [3]. However, it is unclear whether the indirect benefits of such an endeavor outweigh the costs of setting up and operating a city-wide free Wi-Fi network.

For this reason, this publication does not assume public Wi-Fi to necessarily be free for the user. We consider two additional Wi-Fi business models in which, aside from indirect revenues, direct revenues are generated as well. In the freemium model, a user is given the choice to either surf at a limited speed and be shown ads (e.g., injecting an additional header or footer in the visited websites is a typical approach) or to pay for a faster adless connection. Alternatively, using the premium model, users are obligated to pay to get access (though no ads are shown).

Looking at the practical side of Wi-Fi network deployments, multiple parties can be involved: the actor who wants a Wi-Fi network in its venue might pay a second party to perform all technical duties (deployment and maintenance). For example, a small coffee shop might pay a local technician to deploy its network; a city government may depend on the technical expertise of a mobile network operator (MNO) to deploy the network or may even choose to set up long-term collaboration such as a public-private partnership (PPP).

In this paper, we demonstrate how non-cooperative game theory can be used to model the interaction between a technical party (e.g., a wireless network operator) and the owner of a public or semi-public venue when considering the joint deployment of public Wi-Fi. We look into how the relevant network costs (upfront and deployment) and revenues (both direct and indirect) can be modeled for the different pricing models (free, freemium, and premium) and how these costs and revenues can be split between both parties. Finally, we also introduce means of steering the expected outcome of the game in order to end up in the most beneficial outcome possible for all parties.

The remainder of this paper is structured as follows: in section 2, we provide an overview of the existing literature on public Wi-Fi in general, as well as a literature review on non-cooperative games in the context of ICT networks and telecommunications. In section 3, we suggest a methodology for modeling the costs and revenues associated with public Wi-Fi deployment and the game theoretical interplay between the technical party and the venue owner. Section 4 applies this methodology to a public Wi-Fi deployment in a shopping mall and discusses the potential implications on real-life public WiFi projects. In section 5, we give some concluding remarks and propose trajectories for future work.

\section{Literature study and background information}

In this section, we discuss the current state of the art divided in two major sections. The first section (2.1) gives more detail about public Wi-Fi in general. The second section (2.2) focuses upon the game theoretical part and provides the required background for the remainder of the paper. It provides a set of examples in which game theory has been used in both a broader scope of ICT networks as well as for (public) Wi-Fi networks specifically.

\subsection{Public Wi-Fi examples}

While in the early days of customer-facing Wi-Fi, users often had to pay a subscription fee or watch advertisements to gain access to the network, free Wi-Fi in small and medium-sized businesses has been a successful business model for the past decade. Typically, venues with customer-facing Wi-Fi are privately owned. We do however refer to these as public networks, since they are located in semi-public places such as pubs, restaurants, and business venues [4]. Public does not mean unsecured; typically, access keys are shared on-site. This way, the network can mostly be limited to customers who are actually present at the venue, if the key is changed frequently enough. ${ }^{1}$

While customer-facing networks are quite common, city-wide municipal Wi-Fi networks are less so. Municipal Wi-Fi offers a wide range of social and economic benefits such as cost savings in public offices, providing a stimulus to the economy and alleviating the digital divide [5]. Many of these networks had to shut down, however, as they were not economically viable [6].

Next to economic reasons, municipal Wi-Fi offers run into other problems. By offering free or cheap Internet access to inhabitants, local governments enter the highly competitive telecommunications market at ultra-low prices or free of charge. This has even led to municipalities being sued by private Internet Service Providers (ISP) over loss of profit $[2,7]$.

In order to tackle both of these problems at once, we consider non-free municipal Wi-Fi networks which are being rolled out in cooperation with a MNO. As users also generate direct revenues (next to the earlier discussed indirect revenues), the cost of the network can (partly) be covered. In the meantime, the MNO can

\footnotetext{
${ }^{1}$ Adding a security key (e.g., using WPA2) also allows the encryption of all traffic. The impact of this should not be overrated: a possible attacker might still be able to listen in on any plain-text traffic as the security key is publicly known.
} 
benefit from the cooperation instead of suffering from the added competition.

The European Commission (EC) clearly also believes in the indirect economic gains of public Wi-Fi. In 2016, the EC has decided upon the WiFi4EU scheme, which frees up a total of 120 million between 2017 and 2019 to support the roll-out of public Wi-Fi in public spaces such as parks, squares, and public buildings where a free public or a private Wi-Fi hotspot offering does not already exist [8].

\subsection{Introduction to game theory and non-cooperative games}

In the previous section, we provided some additional background about public Wi-Fi networks. This section will do the same for the game theoretical approach used in this publication.

Game theory is the mathematical modeling of both cooperative and non-cooperative scenarios, so-called games. From a high-level point of view, game theory can be split in two categories: cooperative and non-cooperative games. In cooperative games, an analysis is made of how a set of actors can work together most optimally by creating a coalition. In this type of game, the different players will (as the name indicates) cooperate to a common goal.

Next to cooperative games, there are non-cooperative games in which the impact of (market) competition or conflicting objectives is modeled. In this publication, we focus on parallel non-cooperative two-player games. In these games, both players choose their strategy at the same time without knowledge of the chosen strategy of the other actor. Aside from parallel games, there are sequential games. In sequential games actors choose one after the other, taking into account what its opponent has chosen. Sequential games are not further used in this publication.

In non-cooperative games, each combination of strategies (meaning each player has chosen one of its strategies) results in a numerical value for each of the players, the so-called payoff values. Two kinds of games exist: zero-sum games and non-zero-sum games. In a zero-sum game, the sum of the payoffs of both players is equal to zero for each combination of strategies. An outcome for player 1 of plus five will thus have an outcome for player 2 of minus five. Non-zero-sum games are thus games in which the sum of the payoffs is not equal to zero.

How the payoffs are constructed can vary from simple predefined intuitively chosen values to complex mathematical models to which the chosen strategies are the inputs. Payoff values should be modeled carefully, as the outcome of a game theoretical approach depends on them. In the next section, we look more deeply into what makes game theoretical analysis so interesting and how games can be represented using one of the most typical examples (the prisoner's dilemma). Afterwards, a number of examples are provided in which game theory has been used in both a broader scope of ICT networks as well as for Wi-Fi networks in particular.

\subsection{Analysis of non-cooperative games}

Non-cooperative game theory allows for the identification of dominant strategies (strategies that yield a better payoff than any other, independently from the strategies of the other players), Nash equilibria (combinations of strategies where no one player can gain a higher payoff by choosing a different strategy), and Pareto efficient states (combinations of strategies where no player can gain a higher payoff without decreasing any other player's payoff).

Non-cooperative games often end up in a Nash equilibrium which is not a Pareto optimal state, as one or more parties have an incentive to cheat the other(s) by deviating from the Pareto optimal solution. An example for this is the prisoner's dilemma [9] which has a single Nash equilibrium and three Pareto optimal states, none of which equal the Nash equilibrium.

The prisoner's dilemma goes like this: suppose that two partners in crime are independently being questioned by the police (meaning they have no means of communicating with each other). They both have the option to either cooperate (confess) or to remain silent. If they both refuse to cooperate (and thus remain silent), they will both be sentenced with a short jail-time (1 year), lacking sufficient evidence for more severe punishments. If they both cooperate, they both will be sentenced to a medium-length prison sentence (8 years). If one remains silence, and the other cooperates, the one cooperating walks free because of good behavior, while the other one receives the full blame and as result a maximum sentence ( 10 years).

Table 1 shows the typical representation of the outcome of a two-player non-cooperative game; this outcome is called a payoff matrix. The row headers represent the actions of the first player (in this case the first prisoner, P1), and the column headers the representation of the second player (P2). Each cell contains the payoffs of both players, first the payoff of the first player, followed by the second. Additionally, specific cells can be indicated on the matrix to indicate Nash and Pareto equilibria. In this case, there is only one Nash equilibrium, indicating that both prisoners will cooperate (and thus end up in prison for 8 years). This makes sense since, not knowing whether the other one will confess or not, it is always safer to confess and risk a mediumlength sentence than to remain silent and risk a long sentence. This is a so-called dominant strategy. However, this outcome is not the most favorable situation for both. If both had kept silent, they would have been free after just a single year in jail [10].

This makes non-cooperative games very enticing for economists, as it is possible to identify Pareto optimal 
Table 1 Payoff matrix of the prisoners' dilemma upon which both Pareto optimal state (bordered cells) and the Nash equilibrium (bold and underlined) are indicated

P2

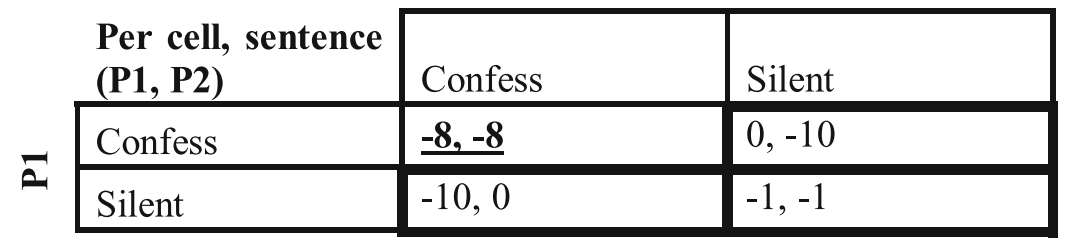

game states, which usually indicates a game state that provides the highest overall payoff. The goal then exists to steer the game by adapting the strategies of one of the players in such a way that one out of the set of outcomes becomes both Nash and Pareto.

\subsubsection{Game theoretical applied to ICT networks}

Generally speaking, game theory has been applied for the optimization of various back-end functions of ICT networks such as resource allocation and routing optimizations [11-13] but also for optimizing power usage [14] as well as for spectrum sharing [15].

More concretely for Wi-Fi networks, non-cooperative game theory has been used for various optimizations and evaluations: e.g., for bandwidth sharing within a single WiFi network [16], for the selection of the optimal network from a set of available Wi-Fi networks [17], for the creation of pricing schemes in order to determine the optimal network to connect to, either Wi-Fi or a competing network (e.g., mobile, WiMax) [18-21], and for reducing mobile volume usage by creating delayed offloading schemes and smart caching via Wi-Fi networks [22-24].

Quite some publications can be found which use game theory for the optimization of ICT networks and Wi-Fi networks in general. The same cannot be said for literature for the game theoretical analysis of public Wi-Fi networks specifically. Prior work discussed the fact that a public-private partnership between the municipality and a private network operator is a viable strategy for public Wi-Fi deployment, but the chances of this happening diminish when competition is added [25]. Other work describes a self-managed scheme that promotes the formation of peer-to-peer free municipal Wi-Fi networks, by opening up underexploited wireless networks [26]. In [27], a study was made to determine a strategic access price (a ticket one has to buy in order to gain access) for public Wi-Fi when offered by a local government and how this is impacted by competition with mobile networks. In [28], the entrance of a new mobile virtual network operator (MVNO) is analyzed. The new player can either lease network resources from an existing $\mathrm{MNO}$ or can fall back to existing Wi-Fi networks. Using game theory, an optimal price setting has been determined. A similar study was made in [29] in which game theory was used to compare $3 \mathrm{G}$ mobile networks with municipal Wi-Fi.

Finally, [30] discussed monetization of Wi-Fi networks either using a freemium (free, though seeing ads) or premium model (paid access). This work focused upon the interaction of multiple parties-a venue owner owning the network, mobile users (MU), advertisers, and advertisement platforms-and states that public Wi-Fi networks “... are capable of generating large revenues through mainly providing one type of $\mathrm{Wi}-\mathrm{Fi}$ access (the premium access or advertising sponsored access), depending on their advertising concentration levels and MU visiting frequencies." Our own publication has a clear link to this but focuses upon the interaction of the venue owner and a mobile owner. In contrast, [30] makes an abstraction of the mobile users and the advertisement platforms. Instead, we focus upon the different costs of different Wi-Fi pricing schemes. A free network will attract more users than a pay-for-access network and need thus be dimensioned accordingly. On top of that, we also consider additional direct and indirect revenue streams next to advertisements.

It is clear that only little work has been done to model the interactions between a technical party and a venue owner for the joint deployment of public Wi-Fi network. These parties, while having a common goal, clearly also have competing goals (each wanting maximal profit). In this publication, we are applying non-cooperative game theory to model exactly these interactions. While other publications mainly apply game theory for the evaluation of different wireless technologies, we are focusing upon different pricing schemes for Wi-Fi networks (free, freemium, and premium). As a result, we are not focusing upon the technical details but rather on the economic side. For this, we have built detailed cost and revenue models in order to estimate the relevant cash flows for both actors, allowing us to make a well-founded game theoretical analysis. Starting from the initial modeled game, we introduce new strategies to steer the outcome of both players to the most optimal solution.

\section{Methods: modeling the payoff values for cooperative Wi-Fi roll-out use cases}

In the previous section, we have introduced what can be learned from a game theoretical approach. Now, we will 
look into how this can be applied to a cooperative Wi-Fi roll-out. As mentioned in section 2.2, in order to construct a payoff matrix, we first have to know which values should be used as the payoff values and consequentially how the payoff values should be modeled and how these are impacted by the different strategies of the players.

For this, we define two players: a MNO and a venue owner (VO). The MNO sees value in a Wi-Fi offer (e.g., for additional revenues or for reducing the load on the mobile network). The VO wants to offer Wi-Fi services to the users in the venue (e.g., a mall owner who wants to attract more customers or a museum that wants to improve the user experience). We have chosen these actors because they are generically applicable and can thus be reused for other use cases. The strategies of these different actors however should be considered use case specific. These will be discussed in section 4; for now, we will focus upon the high-level structure of the payoff values within the game matrix.

As payoff values, we have chosen the net cash flow (i.e., the sum of all revenues minus the sum of all expenditures) after a number of years of operation. In order to do so, we require a detailed cost model for all relevant costs, including capital expenditures (CapEx) and operational expenditures $(\mathrm{OpEx})$, allowing the calculation of the total cost of ownership (TCO) for the Wi-Fi network in the use case at hand which is discussed in section 3.1. Additionally, all direct and indirect revenue streams should be modeled; these are discussed in section 3.2.

In order to make both the payoff of the $\mathrm{MNO}$ and the VO dependent upon both the cost and revenues, we have provided means of splitting these between the players. For the remainder of this publication, we assume the total cost of the Wi-Fi roll-out (both CapEx and $\mathrm{OpEx}$ ) is entirely covered by the network operator, who also receives $100 \%$ of the direct revenue. The VO receives the total indirect revenue. Choosing other cost and revenue split values might be interesting to simulate additional negotiation between both players and other types of use cases (see section 5). Eqs. 1 and 2 show these cost and revenue splits; the definition of the corresponding parameters is given in Table 2 .

$$
\begin{aligned}
& P_{\mathrm{MNO}}=\mathrm{DR}\left(1-s_{r}\right)-\mathrm{TCO}\left(1-s_{c}\right) \\
& P_{\mathrm{VO}}=\mathrm{IR}+\mathrm{DR} \cdot s_{r}-\mathrm{TCO} \cdot s_{c}
\end{aligned}
$$

When actually creating the payoff matrix, it looks like Table 3: the strategies of the MNO are the row headers, the ones of the VO the column headers. Each payoff cell contains 2 values, first the payoff of the MNO followed by the payoff of the VO.

Now that the high-level structure of the payoff values has been introduced, we will give model the values of the
Table 2 High-level parameters used in the payoff functions of the cooperative game

\begin{tabular}{ll}
\hline Parameter & Explanation \\
\hline$P_{\text {MNO }}$ & Payoff mobile network operator \\
$P_{\text {VO }}$ & Payoff venue owner \\
DR & Total direct revenue \\
IR & Total indirect revenue \\
TCO & Total cost of ownership (CapEx + OpEx) \\
$S_{r}$ & Revenue split [0-100\%], default 0\% \\
$S_{C}$ & Cost split [0-100\%], default 0\% \\
\hline
\end{tabular}

parameters. Section 3.1 discussed how the TCO is calculated in, followed by the venue modeling in section 3.2.

\subsection{Equipment cost modeling of Wi-Fi networks}

In order to calculate the TCO of the Wi-Fi network, we have started from a cost model and accompanying detailed bill of materials (BOM) received from the French mobile telecommunications operator Orange. In order to reuse this model and convert it to be useful for the use cases at hand, we categorized the items of the BOM into access point (AP)-driven costs (cost that are directly linked to the number of APs) and additional fixed costs (any additional (network) equipment to get the Wi-Fi network running) as shown in Table 4.

In the BOM, three parameters have been introduced ( $N_{\text {ap }}, N_{\text {switch }}$, and $\left.N_{\text {cable }}\right)$ which will be modeled next in more detail. The first parameter is $N_{\text {ap }}$ which represents the total number of required access points. This is based upon the maximal number of users each AP needs to and is able to handle as well as the area (in $\mathrm{m}^{2}$ ) that needs to be covered combined with the percentage of the area that should be covered (parameter $o$ ). A coverage factor of $80 \%(o=0.8)$ would thus mean $80 \%$ of all total area should be covered. The number of access points needed is formulated as shown in equation Eq. 3; the corresponding parameters are defined in Table 5 . The number of concurrent users and correspondingly the number of APs is directly linked to the different pricing models which will be discussed in section 4.2.

$$
N_{\mathrm{ap}}=\max \left(\frac{u}{u_{\mathrm{AP}}}, \frac{a \cdot o}{a_{\mathrm{AP}}}\right)
$$

Once the total number of the access points is known, we can also calculate the number of Power over Ethernet (PoE) switches required $\left(\mathrm{N}_{\text {switch }}\right)$, which is directly related to the number of APs as shown in Eq. 4. Using PoE, the APs can be provided with electricity without the need of an additional power cable. A minimum of 4 
Table 3 Exemplary payoff matrix of the cooperative game

\begin{tabular}{|c|c|c|c|}
\hline \multirow{2}{*}{\multicolumn{2}{|c|}{$\begin{array}{l}\text { Per cell: } \\
\mathrm{P}_{\mathrm{MNO}(\mathrm{i})}, \mathrm{P}_{\mathrm{VO}(\mathrm{j})}\end{array}$}} & \multicolumn{2}{|l|}{ VO } \\
\hline & & Strategy 1 & Strategy n \\
\hline \multirow{3}{*}{$\begin{array}{l}0 \\
z \\
\Sigma\end{array}$} & Strategy 1 & $\mathrm{P}_{\mathrm{MNO}(0,0)}, \mathrm{P}_{\mathrm{VO}(0,0)}$ & $\mathrm{P}_{\mathrm{MNO}(0, \mathrm{n})}, \mathrm{P}_{\mathrm{VO}(0, \mathrm{n})}$ \\
\hline & $\ldots$ & & \\
\hline & Strategy m & $\mathrm{P}_{\mathrm{MNO}(\mathrm{m}, 0)}, \mathrm{P}_{\mathrm{VO}(\mathrm{m}, 0)}$ & $\mathrm{P}_{\mathrm{MNO}(\mathrm{m}, \mathrm{n})}, \mathrm{P}_{\mathrm{VO}(\mathrm{m}, \mathrm{n})}$ \\
\hline
\end{tabular}

switches are always taken into account for redundancy and for spreading the total traffic load from the APs.

$$
N_{\text {switch }}=\max \left(4, \frac{N_{\mathrm{ap}}}{24}\right)
$$

In section 4, the highest number of APs we are considering is 180 , meaning 8 switches. We suppose the single router (as defined in Table 4) has (a) sufficient ports to connect all switches and (b) sufficient capacity to handle all traffic.

The third and final parameter is $N_{\text {cable }}$ which represents the total length (in meters) of network cable required to connect all APs. To do so, a basic cabling scheme is proposed to estimate the total cable length, using a small set of assumptions:

- The area to be covered is rectangular, with a width of Areaw and a height of Area

- A $n \cdot m$ grid with square cells (with side $s$ ) is layered above the rectangular area and in each cell an AP will be installed. The parameters $n$ and $m$ are chosen in a way so that:

$$
\begin{aligned}
& n \cdot m \approx N_{\text {ap. }} . \\
& \frac{\text { Area }_{\mathrm{L}}}{n} \approx s \\
& \frac{\text { Area }}{m} \approx s
\end{aligned}
$$

\begin{tabular}{|c|c|c|c|}
\hline Item & CapEx & OpEx & Amount \\
\hline \multicolumn{4}{|l|}{ AP-driven costs } \\
\hline Access points (AP) & & & $N_{\text {ap }}$ \\
\hline • Device & $€ 402$ & $10 \%$ & \\
\hline - AP antenna & $€ 272$ & & \\
\hline • Installation & $€ 955$ & & \\
\hline - AP-switch connection & $€ 330$ & & \\
\hline - Floor space and energy consumption & & $€ 427$ & \\
\hline Site maintenance & & $€ 735$ & \\
\hline PoE switch & $€ 1819$ & $10 \%$ & $N_{\text {switch }}$ \\
\hline Switch-router connection & $€ 396$ & & $N_{\text {switch }}$ \\
\hline Ethernet cabling (m) & $€ 1$ & & $N_{\text {Cable }}$ \\
\hline Subsystem installation ${ }^{\mathrm{a}}$ & $€ 210$ & & $N_{\text {switch }}+2$ \\
\hline Electric cabling ${ }^{a}$ & $€ 60$ & & $N_{\text {switch }}+2$ \\
\hline \multicolumn{4}{|l|}{ Fixed costs } \\
\hline Controller & $€ 14,975$ & $10 \%$ & 1 \\
\hline Router & $€ 1819$ & $10 \%$ & 1 \\
\hline Rack & & & 1 \\
\hline • Device & $€ 735$ & $10 \%$ & \\
\hline • Installation & $€ 180$ & & \\
\hline Technical support & $€ 2800$ & & 1 \\
\hline Uplink cost & & $€ 1638.9$ & 2 \\
\hline
\end{tabular}

- Cells are indicated using a Cartesian coordinate system starting in the top left with cell $(0,0)$. In the

Table 4 The reworked bill of materials (BOM) split in AP-driven costs fixed costs

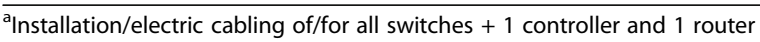


Table 5 Formula parameters for the total number of required access points

\begin{tabular}{ll}
\hline Parameter & Explanation \\
\hline$N_{\text {ap }}$ & The number of required APs \\
$u$ & $\begin{array}{l}\text { The maximum number of users to be connected } \\
\text { at a single point in time } \\
u_{\text {AP }}\end{array}$ \\
a & The number of users supported by a single AP \\
$a_{\text {AP }}$ & The total area to cover \\
0 & The area covered by a single AP \\
\hline
\end{tabular}

middle of each cell an AP is installed, meaning the exact location of the AP in the first cell of this grid is thus positioned at $(0.5 ; 0.5)$ (the $x$ 's in the visualization).

- Switches are installed in 4 locations in the mall from which cable ducts run to the router. These points are chosen in order to minimize the total cable length and are also installed in the middle of the indicated cells (the cells with thick borders in the visualization).

- APs are connected to the closest switch; cables only make right angles.

Taking these assumptions into account, the proposed grid looks like Table 6 , the rectangle has been layered by a 6.5 grid $(n \cdot m)$. Now that this representation is made, the cable length for each AP can easily be estimated, using two basic steps (Eq. 5).

Table 6 Example of an area covered by APs using a $6 \times 5$ grid, with a cell size of $25 \mathrm{~m}$

\begin{tabular}{|c|c|c|c|c|c|c|c|c|}
\hline & & & \multicolumn{6}{|c|}{ Area $_{W}$} \\
\hline & & & $S$ & $s$ & $S$ & $s$ & $S$ & $S$ \\
\hline & & & 0 & 1 & 2 & 3 & 4 & 5 \\
\hline \multirow{5}{*}{ Area $_{H}$} & $S$ & 0 & $\mathrm{X}$ & $X$ & $X$ & X & X & $\mathrm{X}$ \\
\hline & $S$ & 1 & $\mathrm{X}$ & $\mathrm{X}$ & $\mathrm{X}$ & $\mathrm{X}$ & $\mathrm{X}$ & $\mathrm{X}$ \\
\hline & $S$ & 2 & $\mathrm{X}$ & $\mathrm{X}$ & $\mathrm{X}$ & $\mathrm{x}$ & $\mathrm{X}$ & $\mathrm{X}$ \\
\hline & $S$ & 3 & $\mathrm{X}$ & $\mathrm{X}$ & $\mathrm{X}$ & $\mathrm{X}$ & $\mathrm{X}$ & $\mathrm{X}$ \\
\hline & $S$ & 4 & $\mathrm{X}$ & $X$ & $\mathrm{X}$ & $\mathrm{X}$ & $X$ & $\mathrm{X}$ \\
\hline
\end{tabular}

The to-be-covered area with its width (Area $)$ and height $\left(\right.$ Area $_{H}$ ) indicated, and indication of the location of the APS $(x)$, the location of the switches (bordered cells)

$$
\begin{gathered}
\mathrm{CS}=\text { Closest switch point for } \mathrm{AP} \\
\text { Length of cable } \left.=\left|\left(\mathrm{CS}_{\mathrm{x}}-\mathrm{AP}_{\mathrm{x}}\right) * s\right|+\mid \mathrm{CS}_{\mathrm{y}}-\mathrm{AP}_{\mathrm{y}}\right) * s \mid
\end{gathered}
$$

Using this approach, we have calculated the required cable length for each AP as shown in Table 7. Summing all this values results in the total length of cable required.

This approach is a simplification of reality, however. It allows for a basic estimation of the required total cable length when following the listed set of assumptions.

Now that all three variables within the BOM are defined, the TCO can be summarized and structured in 4 major cost groups, CapEx and 3 groups of OpEx costs: site costs, equipment maintenance, and backhauling, as shown in Table 8.

All that remains is taking the sum of the different OpEx costs (Eq. 6), calculating the discounted value of the total OpEx (Eq. 7), and adding it to the CapEx in order to obtain the TCO (Eq. 8). Discounting the values is important as the OpEx costs are spread over multiple years and the time value of money changes. The corresponding parameters are introduced in Table 9.

$$
\begin{aligned}
& c_{\text {OpExUndiscounted }}=c_{\text {OpexOpExSiteCosts }} \\
& +c_{\text {OpexOpExEquipment }} \\
& +c_{\text {OpExbackhauling }} \\
& c_{\mathrm{OpEx}}=\sum_{t=0}^{T} \frac{C_{\text {OpexUndiscounted }}}{(1+r)^{t}} \\
& \mathrm{TCO}=c_{\mathrm{CapEx}}+c_{\mathrm{OpEx}}
\end{aligned}
$$

\subsection{Direct and indirect revenue modeling}

In the previous section, we looked into the TCO. Now, we will have a closer look at the relevant revenues.

Table 7 Required length of cable for each AP to be connected to the closest upload point, with a cell size of $25 \mathrm{~m}$

\begin{tabular}{|c|ccc|c|c|c|}
\hline & 0 & 1 & 2 & 3 & 4 & 5 \\
\hline 0 & 50 & 25 & 50 & 25 & 50 & 75 \\
\cline { 2 - 7 } 1 & 25 & 0 & 25 & 0 & 25 & 50 \\
\hline 2 & 50 & 25 & 50 & 25 & 50 & 75 \\
\hline 3 & 25 & 0 & 25 & 0 & 25 & 50 \\
\hline 4 & 50 & 25 & 50 & 25 & 50 & 75 \\
\hline
\end{tabular}


Table 8 The entire BOM falls down in 4 major cost groups: CapEx and 3 sets of OpEx costs (1 year): site costs, equipment maintenance, and backhauling

\begin{tabular}{|c|c|c|c|}
\hline \multicolumn{4}{|c|}{ Summary Cost Values } \\
\hline Cost Group & Cost components & Value & Amount \\
\hline \multirow[t]{5}{*}{$c_{\text {CapEx }}$} & $C_{\text {CapExAP }}$ & $€ 1959$ & $N_{\text {ap }}$ \\
\hline & $C_{\text {CapExSwitch }}$ & $€ 2215$ & $N_{\text {switch }}$ \\
\hline & $c_{\text {CapExSubsystemAndCabling }}$ & $€ 270$ & $N_{\text {switch }}+2$ \\
\hline & $C_{\text {CapExNetworkCable }}$ & $€ 1$ & $N_{\text {Cable }}$ \\
\hline & $C_{\text {CapExFixed }}$ & $€ 20,509$ & 1 \\
\hline COpExSiteCosts & COpExSiteCosts & $€ 1162$ & $N_{\text {ap }}$ \\
\hline \multirow[t]{3}{*}{ COpExEquipment } & COPEXAP & $€ 40.2$ & $N_{\mathrm{ap}}$ \\
\hline & COpExSwitch & $€ 181.9$ & $N_{\text {switch }}$ \\
\hline & COpExfixed & $€ 1752.9$ & 1 \\
\hline COpExbackhauling & COpExBackhauling & $€ 1638.9$ & 2 \\
\hline
\end{tabular}

Revenues can be categorized in two main categories. On the one hand, there are direct revenues which result from the Wi-Fi offer itself (e.g., ad revenues, analysis of anonymous collected user information, sale of subscription tickets, etc.). On the other hand, there are indirect revenues which result from a positive side effect of the Wi-Fi network (e.g., people might stay longer and thus buy more because there is $\mathrm{Wi}-\mathrm{Fi}$ available). The exact modeling of both types of revenues requires specific approaches. One can easily see that the calculation of ad revenues is different from the calculation of revenue from subscription tickets. Additionally, as indirect revenues are use case specific, we have chosen to discuss both direct and indirect revenues in section 4 and not in a generic way.

\section{Results and discussion}

In the previous sections, we have shown that on a high level, the payoff values are composed of revenues and costs (sections 3.1 and 3.2) and how these can be divided between both parties (section 3). This leaves us only with the actual simulation of the game. Before doing so, we would like to stress that even though a lot of data is clearly based upon the cost model of Orange, we have made a set of assumptions to fill in some of the input

Table 9 Parameters required in order to calculate the discounted TCO

\begin{tabular}{ll}
\hline Parameter & Explanation \\
\hline$C_{\text {OpExUndiscounted }}$ & Total undiscounted OpEx cost \\
COpEx $_{\text {Optal discounted OpEx cost }}$ & Total \\
$C_{\text {CapEx }}$ & Total CapEx cost \\
TCO & Total cost of ownership \\
$T$ & Time horizon of the Wi-Fi network \\
$r$ & Discount rate (\%). \\
\hline
\end{tabular}

values. As assumptions may adversely impact the results, we have chosen to clearly indicate the quality of each input value. For this, we have used a scale between 1 and 5 as shown in Table 10. For the remainder of the paper, all tables with input values will have a column quality which refers to this scale. Do note that the provided input values are assumed for the case at hand and for the time of writing. This means these assumptions might not be applicable in a broader scope and that they should be considered time-sensitive.

In order to demonstrate the game theoretic evaluation of joint Wi-Fi deployment in a realistic scenario, we have built a use case using the earlier discussed cost and revenue models. The setting for this use case is a fictional mall (based on publicly accessible data from Forum des Halles in Paris). We consider a venue with an area of $75,000 \mathrm{~m}^{2}$, an estimated 60,000 visitors during peak hour and a net revenue of 200 million. $^{2}$ We estimate 40 million customers on a yearly basis of which we expect $15 \%$ to connect to the network when a free or freemium network is installed or $2 \%$ in case of premium. An overview of the general use case parameters is provided in Table 11.

In the next sections, we will discuss the different strategies available in the game (section 4.1), followed by the modeled costs and revenues in sections 4.1.1 to 4.1.3.

\subsection{Applying the cost and revenue models to the shopping mall case}

As defined in section 3, we consider 2 players, a MNO and a $\mathrm{VO}$ agreeing to jointly roll-out a Wi-Fi network. In this game, the MNO chooses which kind of Wi-Fi network is offered and has 3 pricing options to choose from: free Wi-Fi (entirely free, no ads), freemium Wi-Fi (either use the network for free with ads, or pay for a better and adless experience), or premium (only paid access, no ads). These pricing models will directly impact the number of users that will connect to the network, and thus the number of APs, switches, and running meter of cable that will be installed (see section 3.1). Besides the amount of equipment installed, the different pricing models do not alter any other technical parameters.

While the MNO chooses which pricing strategy is applied, the VO can offer the MNO a cost split, in other words paying a share of the TCO, ranging from 0 to $100 \%$. As a result, the original payoff formulas (Eqs. 1 and 2) are slightly simplified to their new format as shown in Eqs. 9 and 10, with the corresponding parameters in Table 12.

${ }^{2} \$ 3000$ per square meter was considered a respectable average result in the USA in 2016; applying this to the use case $75.000 \mathrm{~m}^{2} \times \$ 3000 \times 0.9$ $(\$ / €)=202.5$ million euro; rounded to 200 million. Data retrieved from: https://www.statista.com/statistics/741804/leading-us-malls-bysales-per-square-foot/ 
Table 10 Quality scoring overview for the input parameters

\begin{tabular}{|c|c|c|}
\hline Quality & Category & Explanation \\
\hline 1 & Assumption & $\begin{array}{l}\text { This needs to be improved by } \\
\text { gathering realistic data values. }\end{array}$ \\
\hline 2 & $\begin{array}{l}\text { Soft estimate } \\
\text { based on data }\end{array}$ & $\begin{array}{l}\text { Could be improved by constructing } \\
\text { a more elaborate model. }\end{array}$ \\
\hline 3 & $\begin{array}{l}\text { Hard estimate } \\
\text { based on data }\end{array}$ & $\begin{array}{l}\text { The data and underlying models are } \\
\text { sufficiently accurate. }\end{array}$ \\
\hline 4 & $\begin{array}{l}\text { Derived from the } \\
\text { cost model }\end{array}$ & $\begin{array}{l}\text { Based on a hard estimation of costs } \\
\text { by Orange. }\end{array}$ \\
\hline 5 & $\begin{array}{l}\text { Use case } \\
\text { constraint }\end{array}$ & $\begin{array}{l}\text { These values stem directly from the } \\
\text { specifications of the use case (coverage } \\
\text { area, number of visitors, etc.). }\end{array}$ \\
\hline
\end{tabular}

$$
\begin{aligned}
& P_{\mathrm{NMO}}=\mathrm{DR}-\mathrm{TCO} \cdot\left(1-s_{c}\right) \\
& P_{\mathrm{VO}}=\mathrm{IR}-\mathrm{TCO} \cdot s_{c}
\end{aligned}
$$

\subsubsection{Calculating the total cost of ownership (TCO)}

In section 3.1, we have introduced two categories within the equipment cost for the Wi-Fi network: AP-driven and non-AP-driven. In order to know the required number of APs, we use Eq. 3 and feed it with the parameters with the values from Table 13.

This results in a total number of access points $\left(N_{\mathrm{ap}}\right)$ of 180 for free/freemium and 24 for premium in order to cover the entire shopping mall. This value we have linked into the BOM, from which we can deduct the TCO using Eq. 8; the discount rate and time horizon were already introduced in Table 11 . The resulting cost values are listed in Table 14. As discussed in section 3.1, the number of users directly impacts the number of APs and consequentially the entire BOM. As the premium priced network attracts fewer users it will thus be cheaper than the free and freemium priced network.

From Table 14, we can deduct the (discounted) TCO of the Wi-Fi network which, when taking into account a time horizon of 4 years, is nearly $€ 1.2$ million for a free/freemium network and about $€ 200.000$ in case of a premium network. Now that the applicable costs are clear, in the next section the different revenue streams are modeled.
4.1.2 Modeling direct and indirect revenues per Wi-Fi model As said earlier, the revenue streams that should be modeled can be categorized in two categories: direct and indirect revenues. These streams are impacted based upon the chosen Wi-Fi strategy by the MNO as shown in Table 15 . As free and freemium have the same number of users, the same indirect revenues might be expected, this is however not the case. We expect freemium to generate (slightly) less indirect revenues, because an entirely free service (the free pricing model) is more attractive to attract customers to the mall than a service with ads or a paid service (the freemium pricing model). The different revenues streams are discussed in more detail in the next sections.

\subsubsection{Direct revenues: ads and subscription tickets}

The direct revenues consist of two components: on the one hand, there are ad revenues and on the other hand, subscription tickets. Subscription tickets are only relevant in the paid Wi-Fi models (freemium and premium) and are sold for different time spans (hour pass, day pass, year pass (exclusively in premium)). Users who do not buy a subscription ticket under the freemium model generate direct revenue in the form of ad revenue. There are no direct revenues in the free pricing model.

The ad revenue depends upon the total number of non-paying users in the freemium model (Eq. 11); the total revenue for the subscription tickets is the sum of the number of payers per subscription type multiplied with the cost for a single ticket (Eq. 12). As with the OpEx costs, discussed in section 3.1, one has to take into account the time value of money by discounting the total revenues as shown in Eq. 13 and Eq. 14.

$$
\begin{aligned}
& \mathrm{DR}_{\mathrm{ads}}=C_{\text {free }} \cdot U_{\text {free }} \\
& \mathrm{DR}_{\text {tickets }}=\Sigma C_{\mathrm{p}} \cdot U_{\mathrm{p}} \\
& \mathrm{DR}_{\text {undiscounted }}=\mathrm{DR}_{\mathrm{ads}}+\mathrm{DR}_{\text {tickets }} \\
& \mathrm{DR}=\sum_{t=0}^{T} \frac{\mathrm{DR} \mathrm{R}_{\text {undiscounted }}}{(1+r)^{t}}
\end{aligned}
$$

Different numbers of users are expected to buy the different subscription tickets depending upon the chosen

Table 11 General use case parameters

\begin{tabular}{llll}
\hline Input & Explanation & Value & Quality \\
\hline$a$ & The total area of the shopping mall & $75,000 \mathrm{~m}^{2}$ & 5 \\
Cust & Yearly mall visitors & $40,000,000$ & 5 \\
Cust ${ }_{\text {peak }}$ & Visitors during a peak hour & 60,000 & 5 \\
Conn & Percentage of customers who connect to & $15 \%$ (free/freemium) & 5 \\
$T$ & the network & $2 \%$ (premium) & 5 \\
$r$ & Time horizon of the Wi-Fi network & 4 & 4 \\
\hline
\end{tabular}


Table 12 High-level parameters used in the payoff functions of the cooperative game

\begin{tabular}{ll}
\hline Parameter & Explanation \\
\hline$P_{\text {MNO }}$ & Payoff network operator \\
$P_{\text {VO }}$ & Payoff venue owner \\
DR & Total direct revenue \\
IR & Total indirect revenue \\
TCO & Total cost of ownership (CapEx + OpEx) \\
$S_{C}$ & Cost split [0-100\%], proposed by the VO \\
\hline
\end{tabular}

Wi-Fi model as summarized in Table 16. The resulting direct revenue streams are listed in Table 17. Both models result in about the same direct revenue. This may seem odd at first; however, do note that as discussed in the previous section, the TCO for the freemium model is about $€ 1.2$ million, while the TCO of the premium model is much lower with about $€ 200.000$.

\subsubsection{Indirect revenues: side-effects of a public Wi-Fi network}

Next to the direct revenues as discussed in the previous section, we also consider indirect revenues. These do not stem from the provision of the Wi-Fi network itself but are a (desired) extra effect. Since indirect revenues are tied to how attractive users perceive the network, free Wi-Fi generates more indirect revenue than freemium Wi-Fi, which in turn generates more indirect revenue than premium $\mathrm{Wi}$-Fi. A total of 3 different indirect revenue streams considered. Table 18 lists all the parameters which are independent of the chosen Wi-Fi model, while Table 19 lists the dependent ones

- Increase of customers (IDR customers $)$. Offering Wi-Fi to users makes the shopping mall more attractive to enter.

$$
\mathrm{IDR}_{\text {customers }}=\text { Cust }_{\text {incr }} \cdot \text { Sales }
$$

- Increase of shopping time (IDR time$_{\text {the }}$. Customers enjoying the Wi-Fi stay longer at the shopping mall.

$$
\begin{aligned}
& u_{y}=\text { Cust } \cdot \text { Conn } \\
& \operatorname{IDR}_{\text {time }}=u_{y} \cdot t_{\text {incr }} \cdot \text { Sales }_{\text {hourly }}
\end{aligned}
$$

- Increase of sales due to more exposure to ads in the shopping mall (IDR ads). Longer exposure to ads means more likely people are affected by it.

$$
\mathrm{IDR}_{\mathrm{ads}}=u_{y} \cdot \text { Sales } \cdot \text { Sales }_{\mathrm{incr}}
$$

All of these indirect revenues are a benefit for the shops within the mall but not directly for the VO; however, we assume the VO manages to reap a part (10\%) of these benefits because the venue becomes more interesting from a business perspective allowing for higher rental prices. This is shown in Eq. 19; as before, again the revenues are discounted to take into account the time value of money. The resulting values are provided in Table 20.

$$
\begin{gathered}
\mathrm{IDR}_{\text {undiscounted }}=\underset{\cdot}{\left(\mathrm{IDR}_{\text {percentage }}\right.} \\
\mathrm{IDR}=\sum_{t=0}^{T} \frac{\mathrm{IDR}_{\text {undiscounted }}}{(1+r)^{t}}
\end{gathered}
$$

\subsection{Initial version of the game}

Now all costs and revenues have received their value for the use case at hand, it is time to incorporate these in the payoff matrix and combine these correctly with the different strategies of the MNO and the VO. Just a small reminder about the strategies and the game at hand: in the initial version of this game, the MNO chooses how the Wi-Fi-offer will be proposed to the end-user (free, freemium, or premium) while receiving $100 \%$ of the direct revenues. The VO receives the indirect revenues and

Table 13 Formula parameters for the total number of required access points

\begin{tabular}{llll}
\hline Parameter & Explanation & Value & Quality \\
\hline$u$ & The maximum number of users to be connected at & 9000 (free/freemium)1200 (premium) \\
& a single point in time & \\
$u_{\text {AP }}$ & The number of users supported by a single AP & 50 & 5 \\
a & The total area of the shopping mall & $75,000 \mathrm{~m}^{2}$ & 5 \\
0 & Coverage factor & $100 \%$ & 3 \\
$a_{\mathrm{AP}}$ & The area covered by a single AP & $9503 \mathrm{~m}^{2}$ (a circle with a radius of $55 \mathrm{~m}$ ) \\
\hline
\end{tabular}

${ }^{a} 15 \%$ (free/freemium) or $2 \%$ (premium) users connect multiplied by the number of users at peak hour (Cust peak $_{\text {) }}$ 
Table 14 The total cost of ownership calculation shows a cost of over one million euro for a free/freemium Wi-Fi roll-out and two hundred thousand euro for premium

\begin{tabular}{llll}
\hline Parameter & Explanation & Value freemium & Value premium \\
\hline C CapEx $_{\text {COpExSiteCosts }}$ & Total CapEx cost & $€ 397,399$ & $€ 81,855$ \\
COpExEquipment $_{\text {CopExbackhauling }}$ & OpEx for site costs of the APs per year (undiscounted) & $€ 209,160$ & $€ 27,888$ \\
COpEx & OpEx for all non-AP equipment per year (undiscounted) & $€ 10,444.1$ & $€ 3445.30$ \\
TCO & OpEx for backhauling per year (undiscounted) & $€ 3278$ & $€ 3278$ \\
\hline
\end{tabular}

offers to pay a part of the total cost of the network, ranging from 0 to $100 \%{ }^{3}$ We are modeling the interaction between both players as a parallel game; this means both players choose a strategy at the same time, unaware of the other player's strategy.

When simulating the entire game, it results in a game matrix as shown in Table 21 (for information on how to interpret this matrix, see section 3). Within this matrix, a number of cells have been emphasized: the cells with thick borders represent Pareto optimal solutions and the cell in bold and underlined is the single Nash equilibrium. The cells between 20 and $80 \%$ have been removed for brevity, as these follow the same pattern (free and freemium are Pareto, no Nash equilibria are present). In order to calculate Nash and Pareto equilibria, we have applied their definitions as originally defined in [31, 32].

A Nash equilibrium can be defined as follows: it is a stable state in which no participants can gain by a change of strategy as long as all the other participants' strategies remain unchanged. By iterating every cell, we can determine per player individually whether a change of strategy can lead to a better payoff. If no change in strategy of any player can lead to an improvement, the cell is a Nash equilibrium. Doing this for the matrix in Table 21, we see that only for a single cell (the one in the bottom left) none of the players can improve itself by changing only its own strategy. Take for example the cell in the top left $(-1.16,2.06)$ we can see that the MNO can improve its payoff from -1.16 to 8.33 by changing its strategy, making the top left cell not a Nash equilibrium.

Similarly, a Pareto optimal state is a stable state in which no player can improve its payoff without decreasing the payoff of another player. Formulated differently, we say a cell is Pareto dominated if another cell exists

\footnotetext{
${ }^{3}$ This implies a continuous strategy space; however, for the ease of representing the results, we have chosen to discretize the strategies after checking this did not alter the outcome of the game.

${ }^{4}$ In games in which the payoff values have a limited number of possible values, it is possible for two cells to have the same outcome for a player, resulting in the player not having a preference for either. This is not the case in this game.
}

which is as good for all players but strictly better for at least one of the players. ${ }^{4}$ If a cell is not Pareto dominated it is Pareto optimal. By iterating each cell and comparing it with all others, we can validate which cells are Pareto. Taking back the same two examples as before: The bottom left cell $(8.33,0.06)$ is not Pareto as it is dominated by the cell in the middle right $(8.63,0.23)$ which is even better for both players. The top left cell $(-1.16,2.06)$ is Pareto, because no other cell is as good for both players and at least better for one of the players. While both adjacent cells are better for one of the players, they are worse for the other one. These cells are thus not dominating the top left cell. This same reasoning goes for all other cells, making the top left cell Pareto.

When looking at these results, two things should be indicated:

1. The total payoff of each cell (in millions) within a row is identical ${ }^{5}$ : free (0.9), freemium (8.7), and premium (8.36).

2. The Nash equilibrium is not Pareto.

From an outsider's perspective, we can clearly say the Nash equilibrium, the solution that is expected to arise in a market economy, is clearly not the highest yielding set of solutions: from a total payoff point of view, freemium scores better. In more technical terms: the Nash equilibrium is not Pareto optimal because it is Pareto dominated by the multiple combinations (e.g., freemium, $100 \%)$.

\subsection{Adapted version of the game: the introduction of a compensation scheme}

Since the total payoff of freemium is highest of all, in other words a freemium solution will yield the highest benefit for both players combined, such a solution is to be preferred from an outsider's perspective. As the total outcome of the freemium model is larger than the total

\footnotetext{
${ }^{5}$ When looking at the full numbers; within the matrix only rounded numbers are provided for brevity, so minor deviations are possible.
} 
Table 15 The chosen Wi-Fi strategy by the MNO impacts the direct and indirect revenue streams

\begin{tabular}{llll}
\hline Wi-Fi model & Direct revenue & $\begin{array}{l}\text { Expected number of } \\
\text { connecting visitors }\end{array}$ & Expected indirect revenue \\
\hline Free & None & $15 \%$ & Highest \\
Freemium & Ads + subscription tickets & $15 \%$ & Lower \\
Premium & Subscription tickets & $2 \%$ & Lowest \\
\hline
\end{tabular}

Table 16 Direct revenue parameters for the freemium and premium model

\begin{tabular}{|c|c|c|c|c|}
\hline Parameter & Explanation & Value freemium & Value premium & Quality \\
\hline$C_{\text {free }}$ & Number of users not paying and generating revenue via ads & $5,280,000$ & 0 & 1 \\
\hline$U_{\text {free }}$ & Ads revenue per user & $€ 0.1$ & 0 & 1 \\
\hline$C_{p}$ & Number of customers for hour, day or year pass & / & / & / \\
\hline$U_{p}$ & Unit revenue for type of pass, $p \in$ (hour, day, year) & / & / & / \\
\hline Chour & Number of customers for hour pass & 540,000 & 720,000 & 3 \\
\hline Uhour & Unit revenue for an hour pass & $€ 2$ & $€ 2$ & 3 \\
\hline$C_{\text {day }}$ & Number of customers for day pass & 180,000 & 48,000 & 2 \\
\hline$U_{\text {day }}$ & Unit revenue for a day pass & $€ 5$ & $€ 5$ & 2 \\
\hline$C_{\text {year }}$ & Number of customers for year pass & 0 & 32,000 & 2 \\
\hline$U_{\text {year }}$ & Unit revenue for a year pass & 0 & $€ 25$ & 2 \\
\hline
\end{tabular}

Table 17 Generated direct revenues for the freemium and premium Wi-Fi model as calculated by Eqs. 11-14

\begin{tabular}{llll}
\hline Parameter & Explanation & Freemium & Premium \\
\hline$D R_{\text {ads }}$ & Undiscounted revenue from ads per year & $€ 528,000$ & 0 \\
$D R_{\text {tickets }}$ & Undiscounted revenue from subscription tickets per year & $€ 1,980,000$ & $€ 2,480,000$ \\
$D R_{\text {undiscounted }}$ & Total undiscounted direct revenue per year & $€ 2,508,000$ & $€ 2,480,000$ \\
$D R$ & Total discounted revenues (for 4 years) & $€ 8,625,012$ & $€ 8,528,720$
\end{tabular}

Table 18 Indirect revenue parameters shared for all three Wi-Fi models

\begin{tabular}{|c|c|c|c|}
\hline Parameter & Explanation & Value & Quality \\
\hline Sales & Yearly total revenue of the mall & $€ 240,000,000$ & 5 \\
\hline Cust & Yearly mall visitors & $40,000,000$ & 5 \\
\hline Saleshourly & Hourly net revenue gained from customers who spend longer time inside due to Wi-Fi & 8 & 1 \\
\hline$t_{\text {incr }}$ & Time that a Wi-Fi user spends longer in the mall (compared to non-users) & $15 \mathrm{~min}$ & 1 \\
\hline Salesincr & The increase of sales due to more exposure to ads in the shopping mall & $3 \%$ & 5 \\
\hline
\end{tabular}

anderlying reasoning: people might stay a little longer in the mall $(15 \mathrm{~min})$, e.g., to look up where to go next, while drinking a coffee worth 2 euro

Table 19 Indirect revenue parameters with distinct values for all three Wi-Fi models

\begin{tabular}{|c|c|c|c|c|c|}
\hline Parameter & Explanation & Value free & Value freemium & Value premium & Quality \\
\hline Custincr & Increase of customers due to availability of Wi-Fi & $2 \%$ & $1 \%$ & $0 \%$ & 5 \\
\hline Conn & Percentage of customers who connect to the network & $15 \%$ & $15 \%$ & $2 \%$ & 5 \\
\hline$u_{y}$ & The number of users that connect to the network per year & $6,000,000$ & $6,000,000$ & 120,000 & 5 \\
\hline
\end{tabular}


Table 20 Indirect revenue streams for different Wi-Fi models

\begin{tabular}{|c|c|c|c|c|}
\hline Parameter & Explanation & Value free & Value freemium & Value premium \\
\hline IDR customer & Undiscounted revenue by increase in customers per year & $€ 4,800,000$ & $€ 2,400,000$ & 0 \\
\hline IDR & Undiscounted revenue by increased sales longer time spent in mall per year & $€ 120,000$ & $€ 120,000$ & $€ 16,000$ \\
\hline IDR ads & Undiscounted revenue by increased sales through targeted ads per year & $€ 1,080,000$ & $€ 1,080,000$ & $€ 144,000$ \\
\hline IDR percentage & Percentage of the estimated revenues the venue owner can charge. & $10 \%$ & $10 \%$ & $10 \%$ \\
\hline$I D R_{\text {undiscounted }}$ & Total indirect revenues per year & $€ 600,000$ & $€ 360,000$ & $€ 16,000$ \\
\hline IDR & Total discounted indirect revenues (for 4 years) & $€ 2,063,400$ & $€ 1,238,040$ & $€ 55,024$ \\
\hline
\end{tabular}

outcome of the premium model, a redistribution of the payoffs between both actors is possible.

The redistribution is as follows: the VO proposes next to taking over a part of the TCO (just as before) to pay an additional fee equal to a percentage of the premium profits. This way, the MNO receives the entire direct revenues of the freemium model and a part of what would have been earned if chosen for the premium model.

Which compensation percentages could work can be determined from Fig. 1; in this figure, we have plotted the difference in profit for the MNO and VO when compared to the premium model (positive means more profit). The interesting area in this figure is where both lines are positive, meaning both actors will thus obtain more profit from the freemium model than the premium model. This implies that the VO can convince the MNO to choose for a freemium solution if he offers at least $10.5 \%$ additional compensation $(10.5 \%$ of the total revenues in the premium model). Additionally, the VO cannot (should not) offer more than $14 \%$ compensation, as that would make paying the compensation to end up in the freemium model less interesting than the premium model from his point of view.

In this figure, we assume a cost sharing of $0 \%$ is considered, the range of possible compensation percentages will obviously differ at other cost sharing percentage: higher cost sharing means less profit for the VO, meaning less profit which can be used for compensation. Since the Nash equilibrium is situated at $0 \%$ cost sharing, discussing compensation at other percentages than $0 \%$ in details yields no additional knowledge.

As any percentage between 10.5 and $14 \%$ will yield the desired effect, we have chosen arbitrarily to use $11 \%$. The proposed changes in the game have been visualized in Table 22; the first strategy of the VO ( $0 \%$ cost share) is extended to $0 \%$ cost share $+11 \%$ compensation; $11 \%$ of the revenues of the premium model is equal to 0.949 million (the direct and indirect revenues were discussed in Table 17 and Table 20). All 11 strategies of the mall owner $(0-100 \%$ in $10 \%$ increments) can be adapted to include the proposed compensation scheme.

This kind of adaption to the game reflects to negotiations between $\mathrm{MNO}$ and $\mathrm{VO}$ in real life, e.g., the MNO could state "We can agree with a Freemium pricing model, as long as it generates us as much profit as a Premium pricing strategy". It is then up to the VO to see whether and how this is possible. In this case, we have chosen to work out an additional compensation as a percentage of the premium revenue; absolute compensations or other cost sharing strategies would be possible as well.

When recalculating the game with these newly proposed strategies, we see the single Nash equilibrium has moved up to the freemium row; this way, the expected outcome of the game (Nash) corresponds with a Pareto optimal solution. In other words, the game has effectively been steered to the optimal solution, which was the original

Table 21 Extract of the matrix of the initial game, expressed in million: bordered cells are Pareto, bold underlined cells are Nash

\begin{tabular}{|c|c|c|c|c|c|c|c|c|c|c|c|c|c|}
\hline \multirow{3}{*}{\multicolumn{2}{|c|}{$\begin{array}{l}\text { Per cell: } \\
\text { P }_{\text {MNO }}, \mathbf{P}_{\text {Vo }} \\
\text { Free }\end{array}$}} & \multicolumn{12}{|c|}{ VO } \\
\hline & & \multicolumn{2}{|c|}{$0 \%$} & \multicolumn{2}{|c|}{$10 \%$} & \multicolumn{2}{|c|}{$20 \%$} & \multicolumn{2}{|c|}{$80 \%$} & \multicolumn{2}{|c|}{$90 \%$} & \multicolumn{2}{|c|}{$100 \%$} \\
\hline & & -1.16 & 2.06 & -1.05 & 1.95 & -0.93 & 1.83 & -0.23 & 1.13 & -0.12 & 1.02 & 0.00 & 1.19 \\
\hline \multirow{2}{*}{$\stackrel{0}{Z}$} & Freemium & 7.46 & 1.24 & 7.58 & 1.12 & 7.69 & 1.01 & 8.39 & 0.31 & 8.51 & 0.19 & 8.63 & 0.23 \\
\hline & Premium & $\underline{8.33}$ & $\underline{0.06}$ & 8.35 & 0.03 & 8.37 & 0.01 & 8.49 & -0.11 & 8.51 & -0.13 & 8.53 & -0.14 \\
\hline
\end{tabular}




\section{Effect of different compensation percentages (at $0 \%$ cost sharing)}

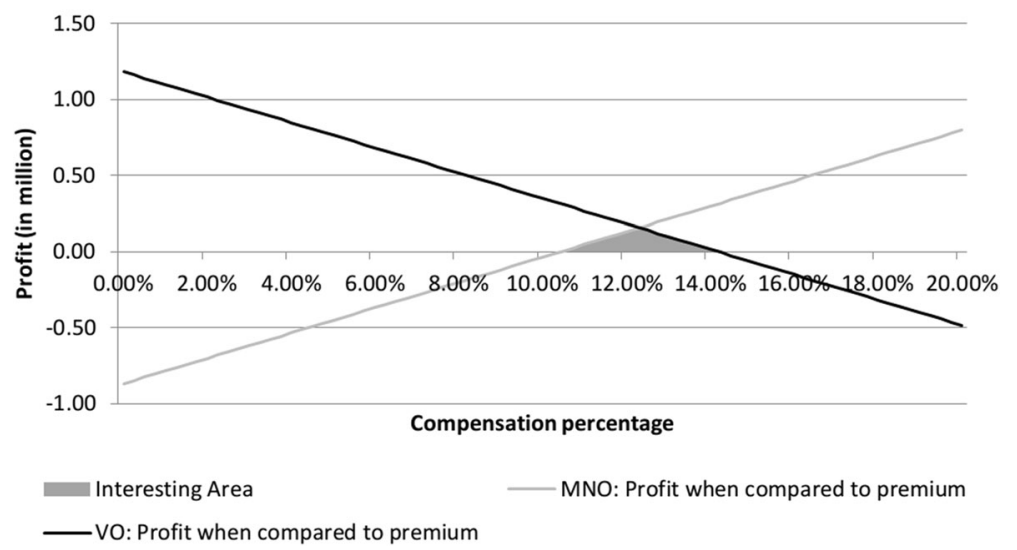

Fig. 1 Effect of different compensation percentages when comparing the profit of the MNO with the premium model. The interesting area is where both lines are positive, meaning both actors will obtain more profit from the freemium model than the premium model. This implies that the VO can convince the MNO to choose for a freemium solution if he offers at least $10.5 \%$ additional compensation ( $10.5 \%$ of the total revenues in the premium model)

intent. The adapted outcome of the game is provided in Table 23; the cells between 0 and $80 \%$ have been removed for brevity, as these follow the same pattern (free and freemium are Pareto, no Nash equilibria are present).

\section{Conclusion}

In this paper, we have demonstrated how to model the interactions between a MNO and a VO in a twoplayer non-cooperative non-zero-sum game with the goal of deploying a public Wi-Fi network. For this we have modeled both the cost of the deployment of the Wi-Fi network (CapEx and OpEx) as well as the direct and indirect revenues. For the cost information, we have used a simplified version of a cost model provided by Orange, where the number of users connecting to the Wi-Fi network and the coverage area are the main cost drivers.

The considered use case describes the deployment of public Wi-Fi in a shopping mall in which the MNO chooses a business model (free, freemium, premium), while the VO (in this case, the shopping mall owner) proposes to take over a part of the network cost $(0-$ $100 \%)$. Starting from this game, we have seen that the expected outcome of the game is a premium Wi-Fi offering, a solution which is not Pareto optimal. However, when modeling the outcome of expected negotiations between MNO and VO in real life, we end up with a situation where the $\mathrm{VO}$ pays a compensation to the $\mathrm{MNO}$ to choose a freemium Wi-Fi solution. By agreeing to this compensation, the payoff of the MNO becomes as good for freemium as for premium, while in the meantime the VO manages to increase his payoff even when paying the compensation. This way, the game ends up in a solution which is both Pareto optimal and a Nash equilibrium.

The purpose of this paper is to describe the methodology of how to model the outcome of a collaborative public Wi-Fi deployment, not to give an exact economic

Table 22 Example of how the new strategies of the MNO might look

\begin{tabular}{|c|c|c|c|c|c|}
\hline \multirow[b]{2}{*}{$\begin{array}{l}\text { Per cell: } \\
\mathbf{P}_{\mathrm{MNO}}, \mathbf{P}_{\mathrm{VO}}\end{array}$} & \multicolumn{5}{|c|}{ VO } \\
\hline & \multicolumn{2}{|c|}{$0 \%$} & & \multicolumn{2}{|c|}{$\begin{array}{c}0 \%+10 \% \\
\text { compensation }\end{array}$} \\
\hline Free & -1.16 & 2.06 & \multirow{3}{*}{$+0.949,-0.949$} & -1.16 & 2.06 \\
\hline Freemium & 7.46 & 1.24 & & 8.41 & 0.29 \\
\hline Premium & 8.33 & 0.06 & & 8.33 & 0.06 \\
\hline
\end{tabular}


Table 23 Extract of the matrix of the adapted game, expressed in million: bordered cells are Pareto, bold underlined cells are Nash; in the adapted version the Nash has become Pareto

\begin{tabular}{|c|c|c|c|c|c|c|c|c|c|c|c|c|c|}
\hline & & & & & & & & & & & & & \\
\hline & $\begin{array}{l}\text { Per cell: } \\
\mathbf{P}_{\mathrm{MNO}}, \mathbf{P}_{\mathrm{VO}}\end{array}$ & & & & & & & & & & & & \\
\hline & Free & -1.16 & 2.06 & -1.05 & 1.95 & -0.93 & 1.83 & -0.23 & 1.13 & -0.12 & 1.02 & 0.00 & 0.90 \\
\hline$\Sigma$ & Freemium & 8.41 & $\underline{0.29}$ & 8.53 & 0.17 & 8.64 & 0.06 & 9.34 & -0.64 & 9.46 & -0.76 & 9.57 & -0.87 \\
\hline
\end{tabular}

prediction of a real-life use case. We have proposed a methodological approach for modeling costs and revenues that is general enough to remain valid with different input values. However, the actual outcome of the model is highly sensitive to the input data. In order to make reliable predictions about real-life use cases, one needs to obtain reliable input values through market research and analysis of the technical constraints of the specific use case at hand. In the case of municipal Wi-Fi, both the willingness to pay and the corresponding number of paying customers, as well as the indirect benefits from the network itself should be well researched or modeled, e.g., by constructing a more detailed bottomup model of such benefits [33].

Multiple tracks are possible for future work: a first step could be to further refine the models by including more technical details. We currently do not assume that data offloading has a large impact on a mobile network operator's revenue in enclosed areas such as the shopping malls. Adding the indirect effects of data offloading might change the network operator's outcome, for better or worse. On the one hand, offloading decongests the cellular network, but on the other hand, the network operator might lose revenue when subscribers choose to transmit their mobile data over a Wi-Fi network instead of the cellular network.

Additionally, femtocells could be added in this comparison as these also help in decongesting the network and do not have the risk of reducing the mobile revenues; in such a case, the comparison between Wi-Fi and femtocells could be made. Other technical parameters such as the location of APs could be included.

Next to a more thorough technical modeling, a validation of the input values could be interesting. This could either be performed using a sensitivity analysis, testing the impact of, e.g., the number of concurrent users and the cost of specific equipment. Another approach could be constructing a methodological framework which allows companies to make qualified estimates of the considered input values.

\section{Abbreviations}

AP: Access point; BOM: Bill of materials; CapEx: Capital expenditures;

EC: European Commission; MNO: Mobile network operator; MU: Mobile users;
OpEx: Operational expenditure; PPP: Public-private-partnership; TCO: Total cost of ownership; VO: Venue owner

\section{Acknowledgements}

Not applicable

\section{Authors' contributions}

$J S$ is the main writer of this publication. AB and MC carried out the cost and revenue modeling. SV and DC contributed to the used models and reviewed the publication internally on multiple occasions. All authors read and approved the final manuscript.

\section{Funding}

Not applicable.

\section{Availability of data and materials}

All input data for this study are included in this published article; the resulting raw output is available from the corresponding author on reasonable request.

\section{Competing interests}

The authors declare that they have no competing interests.

\section{Author details}

${ }^{1}$ IDLab, Department of Information Technology, Ghent University-imec, Technologiepark-Zwijnaarde 15, 9052 Ghent, Belgium. ${ }^{2}$ Orange Gardens, 44 Avenue de la République, CS 50010, 92326 Chatillon Cedex, France.

Received: 23 August 2018 Accepted: 11 September 2019

Published online: 30 October 2019

\section{References}

1. Cisco, "Cisco VNI Mobile Forecast (2016-2021)," 06 February 2017. [Online]. Available: http://www.cisco.com/c/en/us/solutions/collateral/serviceprovider/visual-networking-index-vni/mobile-white-paper-c11-520862.html. [Accessed 07 Feb 2017]

2. J. Gibbons, S. Ruth, Municipal Wi-Fi: big wave or wipeout? IEEE Internet Comput. 10(3), 66-71 (2006)

3. M.B. Weiss, K. Huang, To Be or Not to Be: A Comparative Study of City-Wide Municipal Wifi in the US (2007)

4. K.N. Hampton, N. Gupta, Community and social interaction in the wireless city: wi-fi use in public and semi-public spaces. New Media Soc. 10(6), 831-850 (2008)

5. F. Bar, N. Park, Municipal Wi-Fi networks: the goals, practices, and policy implications of the US case. Commun. Strateg. 61, 107-126 (2005)

6. T.W. Kaiser, S. Verbrugge, M. Van der Wee, D. Colle, An Overview of Different Business Models for Public Wi-Fi and their Implications on Indirect Revenue (2017)

7. J. Freeman, in E-Government Success Around the World: Cases, Empirical Studies, and Practical Recommendations (pp. 235-258). Local e-government and citizen participation: Case studies from Australia and Italy (IGI Global, 2013)

8. European Commission, "WiFi4EU - Free Wi-Fi for Europeans," 31 January 2018. [Online]. Available: https://ec.europa.eu/digital-single-market/en/ policies/wifi4eu-free-wi-fi-europeans. [Accessed 5 Feb 2018]

9. A.W. Tucker, P.D. Straffin Jr., The mathematics of Tucker: a sampler. Two-Year Coll. Math. J. 14(3), 228-232 (1983) 
10. A. Rapoport, A.M. Chammah, C.J. Orwant, Prisoner's Dilemma: A Study in Conflict and Cooperation, vol 165 (University of Michigan press, 1965)

11. Z. Han, D. Niyato, W. Saad, T. Başar, A. Hjørungnes, Game Theory in Wireless and Communication Networks: Theory, Models, and Applications (Cambridge University Press, 2012)

12. X. Chen, Y. Chen, W. Liao, Z. Zhou, B. Gu, X. Wang, Optimal pricing strategy for resource allocation in $5 \mathrm{G}$ heterogeneous cellular networks. Trans. Emerg. Telecommun. Technol. 29(10), e3437 (2018)

13. D.E. Charilas, A.D. Panagopoulos, A survey on game theory applications in wireless networks. Comput. Netw. 54(18), 3421-3430 (2010)

14. A.Y. Al-Zahrani, F.R. Yu, An energy-efficient resource allocation and interference management scheme in green heterogeneous networks using game theory. IEEE Trans. Veh. Technol. 65(7), 5384-5396 (2016)

15. Q. Ni, R. Zhu, Z. Wu, Y. Sun, L. Zhou, B. Zhou, Spectrum allocation based on game theory in cognitive radio networks. JNW 8(3), 712-722 (2013)

16. Z. Fang, B. Bensaou, in IEEE infocom. Fair bandwidth sharing algorithms based on game theory frameworks for wireless ad hoc networks, vol 2 (INSTITUTE OF ELECTRICAL ENGINEERS INC (IEEE), 2004), pp. 1284-1295

17. Nguyen-Vuong, Q. T., Ghamri-Doudane, Y., \& Agoulmine, N. (2008). On utility models for access network selection in wireless heterogeneous networks. In Network Operations and Management Symposium, 2008. NOMS 2008. IEEE (pp. 144-151).

18. L. Yang, W. Lou, Evolution and competition, a game theoretical analysis of heterogeneous wireless networks in unlicensed spectrum. IEEE Access 6 14347-14356 (2018)

19. D. Niyato, E. Hossain, Wireless broadband access: wimax and beyondintegration of wimax and wifi: optimal pricing for bandwidth sharing. IEEE Commun. Mag. 45(5), 140-146 (2007)

20. M. Bennis, M. Simsek, A. Czylwik, W. Saad, S. Valentin, M. Debbah, When cellular meets WiFi in wireless small cell networks. IEEE Commun. Mag. 51(6), 44-50 (2013)

21. S. Shin, M.B. Weiss, Analysis of mobile broadband competition: $3 \mathrm{G}$ vs. WiFi. Int. J. Mob. Commun. 8(5), 586-601 (2010)

22. S. Cai, L. Duan, J. Wang, S. Zhou, R. Zhang, in Communications (ICC), 2015 IEEE International Conference on (pp. 3388-3393). Incentive mechanism design for delayed WiFi offloading (IEEE, 2015)

23. N. Cheng, N. Lu, N. Zhang, X. Zhang, X.S. Shen, J.W. Mark, Opportunistic WiFi offloading in vehicular environment: a game-theory approach. IEEE Trans. Intell. Transp. Syst. 17(7), 1944-1955 (2016)

24. K. Hamidouche, W. Saad, M. Debbah, in Modeling and Optimization in Mobile, Ad Hoc, and Wireless Networks (WiOpt), 2014 12th International Symposium on (pp. 569-574). Many-to-many matching games for proactive social-caching in wireless small cell networks (IEEE, 2014)

25. M. Tahon, B. Lannoo, J. Van Ooteghem, K. Casier, S. Verbrugge, D. Colle, et al., Municipal support of wireless access network rollout: a game theoretic approach. Telecommun. Policy 35(9-10), 883-894 (2011)

26. E.C. Efstathiou, G.C. Polyzos, in World of Wireless Mobile and Multimedia Networks, 2005. WoWMoM 2005. Sixth IEEE International Symposium on a (pp. 502-506). A self-managed scheme for free citywide wi-fi (IEEE, 2005)

27. S. Shin, J.E. Tucci, A study of e-government infrastructure through WiFi implementation: strategic pricing to recover costs. Electron. Gov Int. J. 6(3), 282-294 (2009)

28. B. Khalifa, A. Benhamiche, A. Simonian, M. Bouillon, in IFIP Networking 2018. Profit and strategic analysis for MNO-MVNO partnership (2018)

29. J.P. Shim, S. Shin, M.B. Weiss, in Wireless Telecommunications Symposium, 2007. WTS 2007 (pp. 1-6). Wireless internet competition: Municipal wireless vs. $3 G$ mobile service (IEEE, 2007, April)

30. H. Yu, M.H. Cheung, L. Gao, J. Huang, Public Wi-Fi monetization via advertising. IEEE/ACM Tran. Netw. 25(4), 2110-2121 (2017)

31. Nash, J. (1951). Non-cooperative games. Annals of Mathematics, 54(2), second series, 286-295. doi:https://doi.org/10.2307/1969529

32. Pareto, V., \& Schwier, A. S. (1927). Manual of Political Economy Tr. by Ann S. Schwier. Macmillan, London

33. M. Van der Wee, S. Verbrugge, B. Sadowski, M. Driesse, M. Pickavet, Identifying and quantifying the indirect benefits of broadband networks for e-government and e-business: a bottom-up approach. Telecommun. Policy 39(3), 176-191 (2015)

\section{Publisher's Note}

Springer Nature remains neutral with regard to jurisdictional claims in published maps and institutional affiliations.

\section{Submit your manuscript to a SpringerOpen ${ }^{\circ}$ journal and benefit from:}

- Convenient online submission

- Rigorous peer review

- Open access: articles freely available online

- High visibility within the field

- Retaining the copyright to your article

Submit your next manuscript at $\boldsymbol{\sim}$ springeropen.com 